\title{
A CONCENTRATION OF CENTIMETER-SIZED GRAINS IN THE OPHIUCHUS IRS 48 DUST TRAP
}

\author{
N. van der Marel ${ }^{1}$, P. Pinilla ${ }^{1}$, J. Tobin $^{1,3}$, T. van Kempen ${ }^{1}$, S. Andrews ${ }^{2}$, L. Ricci $^{2}$, And T. Birnstiel ${ }^{2}$ \\ ${ }^{1}$ Leiden Observatory, P.O. Box 9513, 2300 RA Leiden, The Netherlands; nmarel@ strw.leidenuniv.nl \\ ${ }^{2}$ Harvard-Smithsonian Center for Astrophysics, 60 Garden Street, Cambridge, MA 02138, USA \\ Received 2015 March 25; accepted 2015 August 1; published 2015 August 24
}

\begin{abstract}
Azimuthally asymmetric dust distributions observed with the Atacama Large Millimeter/submillimeter Array (ALMA) in transition disks have been interpreted as dust traps. We present Very Large Array Ka band (34 GHz or $0.9 \mathrm{~cm})$ and ALMA Cycle 2 Band $9(680 \mathrm{GHz}$ or $0.45 \mathrm{~mm})$ observations at a 0 ". 2 resolution of the Oph IRS 48 disk, which suggest that larger particles could be more azimuthally concentrated than smaller dust grains, assuming an axisymmetric temperature field or optically thin $680 \mathrm{GHz}$ emission. Fitting an intensity model to both data demonstrates that the azimuthal extent of the millimeter emission is $2.3 \pm 0.9$ times as wide as the centimeter emission, marginally consistent with the particle trapping mechanism under the above assumptions. The $34 \mathrm{GHz}$ continuum image also reveals evidence for ionized gas emission from the star. Both the morphology and the spectral index variations are consistent with an increase of large particles in the center of the trap, but uncertainties remain due to the continuum optical depth at $680 \mathrm{GHz}$. Particle trapping has been proposed in planet formation models to allow dust particles to grow beyond millimeter sizes in the outer regions of protoplanetary disks. The new observations in the Oph IRS 48 disk provide support for the dust trapping mechanism for centimeter-sized grains, although additional data are required for definitive confirmation.
\end{abstract}

Key words: instabilities - planet-disk interactions - planets and satellites: formation - protoplanetary disks

\section{INTRODUCTION}

Studies of transitional disks-protoplanetary disks with inner holes in their dust distribution-are revolutionizing our understanding of planet formation (see the review by Espaillat et al. 2014). High spatial resolution Atacama Large Millimeter/ submillimeter Array (ALMA) observations reveal not only the dust cavities, but also azimuthal asymmetries (Casassus et al. 2013; Fukagawa et al. 2013; van der Marel et al. 2013; Pérez et al. 2014) and gas still present inside the dust cavities (Casassus et al. 2013; van der Marel et al. 2013; Bruderer et al. 2014; Zhang et al. 2014; Perez et al. 2015; van der Marel et al. 2015a). Dust trapping has been suggested as a solution for the radial drift problem (Whipple 1972; Weidenschilling 1977; Brauer et al. 2008) which prevents dust grains from growing beyond millimeter-sizes in the outer disk. Trapping facilitates the crucial steps in dust growth toward the formation of planetesimals (and therefore planets). Because dust continuum emission is dominated by grains with sizes up to three times the observing wavelength (Draine 2006), different continuum wavelengths probe different particle sizes. Since particle trapping depends on particle size, disk turbulence, and the pressure gradient profile, multi-wavelength observations are required to confirm the trapping scenario and constrain disk parameters such as viscosity (e.g., Birnstiel et al. 2013, and references therein).

The Oph IRS 48 transition disk exhibits a highly asymmetric structure in the $0.45 \mathrm{~mm}$ dust continuum. The continuum emission is generated by millimeter-sized grains gathered in a peanut-shaped structure, spanning less than a third of the disk ring azimuth; the peak of this structure is $>100 \times$ higher than the opposite side of the ring (van der Marel et al. 2013). However, the gas traced by ${ }^{12} \mathrm{CO} J=6-5$ line emission has an axisymmetric disk distribution down to $20 \mathrm{AU}$ in radius (Bruderer et al. 2014), confirmed by CO isotopologue

\footnotetext{
${ }^{3}$ VENI fellow.
}

observations of the same disk (van der Marel et al. 2015b). Likewise, the thermal mid-infrared and near-infrared scattered light emission, tracing small micrometer-sized grains, suggest a ring-like structure although variations along the ring cannot be seen due to high optical depth (Geers et al. 2007; Follette et al. 2015). A separation between large and small grains/gas indicates trapping of the large grains in a pressure trap (Barge \& Sommeria 1995; Klahr \& Henning 1997; Brauer et al. 2008; Pinilla et al. 2012). Azimuthal traps may result from vortices, which can be due to instabilities such as the Rossby wave or baroclinic instability (e.g., Lyra \& Lin 2013; Raettig et al. 2013; Fung et al. 2014; Flock et al. 2015). A vortex locally increases the gas pressure over a limited radial and azimuthal extent. In the outer disk, millimeter-sized particles will then drift toward this pressure maximum and get trapped in the azimuthal direction (e.g., Ataiee et al. 2013; Zhu \& Stone 2014), which can explain the observed features in IRS 48. Inside dust traps, the dust particles continue to grow to the maximum grain size permitted by the fragmentation barrier (Birnstiel et al. 2010), which in the outer disk corresponds to centimeter-sizes (e.g., Pinilla et al. 2012). Further growth to planetesimal sizes can occur by streaming instabilities (Johansen et al. 2009) or by taking mass transfer effects into account (Windmark et al. 2012).

The trapping depends on the particle size: small dust particles are strongly coupled to the gas and thus follow the gas distribution, while larger particles (traced at longer wavelengths) are less coupled and therefore feel the drag force toward the pressure maximum, resulting in a more spatially concentrated distribution of these particles, both radially and azimuthally. Both the trapping concentration and the maximum particle size in the trap are dependent on the turbulence and the gas surface density (Pinilla et al. 2015). In this paper, we present spatially resolved observations of Oph IRS 48 at both millimeter and centimeter wavelengths, taken with the ALMA and the Karl G. Jansky Very Large Array (VLA). The 
Table 1

Image Properties

\begin{tabular}{|c|c|c|c|c|c|c|c|}
\hline Telescope & $\begin{array}{l}\text { Frequency } \\
(\mathrm{GHz})\end{array}$ & $\begin{array}{l}\text { Bandwidth } \\
\text { (GHz) }\end{array}$ & Beam Size & Beam PA & $\begin{array}{l}\text { Flux } \\
(\mathrm{mJy})\end{array}$ & $\begin{array}{l}\text { Peak } \\
\left(\mathrm{mJy}_{\text {beam }}{ }^{-1}\right)\end{array}$ & $\begin{array}{l}\mathrm{rms} \\
\left(\mathrm{mJy} \text { beam }^{-1}\right)\end{array}$ \\
\hline ALMA & 680 & 4.7 & $0 " .19 \times 0 " .15$ & $79^{\circ}$ & 1000 & 190 & 0.6 \\
\hline VLA & 34 & 8.0 & $0.46 \times 0 . .26$ & $21^{\circ}$ & $251 \times 10^{-3}$ & $138 \times 10^{-3}$ & $3.5 \times 10^{-3}$ \\
\hline
\end{tabular}

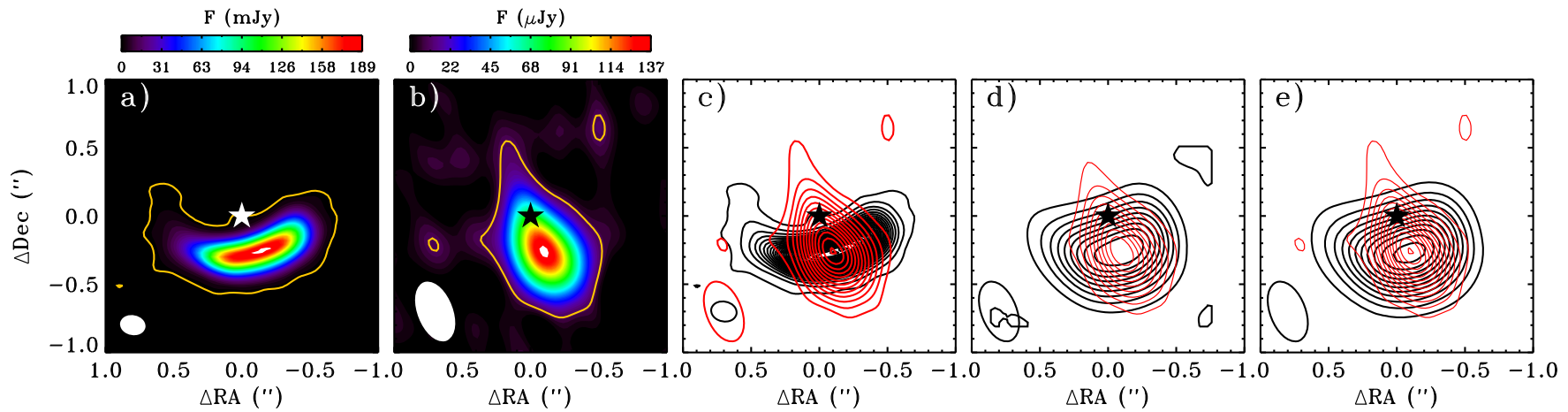

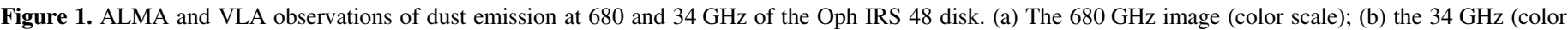

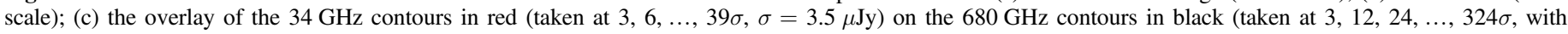

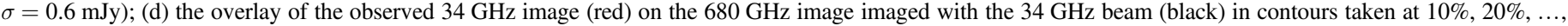

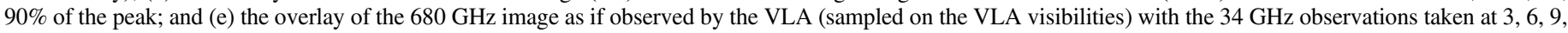
$12, \ldots \sigma$, with $\sigma=3.5 \mu \mathrm{Jy}$. Ellipses indicate the beam size listed in Table 1 .

distributions of dust emission at $450 \mu \mathrm{m}$ and $0.9 \mathrm{~cm}$ are compared and we aim to provide confirmation of the dust trap scenario in a transitional disk. Section 2 describes the observation setup and calibration process, Section 3 presents the images and the derived intensity profile and Section 4 discusses the interpretation of the different morphologies and possible implications for the dust trapping scenario.

\section{OBSERVATIONS}

Observations of Oph IRS 48 at $34 \mathrm{GHz}(9 \mathrm{~mm})$ were obtained using the VLA in the $\mathrm{CnB}$ and $\mathrm{B}$ configurations in 2015 January-February as part of program 14B-115, with baselines ranging from 75 to $8700 \mathrm{~m}$. The spectral windows were configured for a maximum possible continuum bandwidth of $8 \mathrm{GHz}$ centered at $34 \mathrm{GHz}$ ( $\mathrm{Ka}$ band) in dual polarization, using 64 spectral windows of $128 \mathrm{MHz}$ each, with 3-bit sampling. Due to the low declination, the source was observed in three scheduling blocks of $2.75 \mathrm{hr}$ with $1.25 \mathrm{hr}$ on source in each block. The bandpass was calibrated using J1517-2422 (in the third block J1924-2914 was used instead), the absolute flux was calibrated using 3C286 and J1625-2527 was used as a gain calibrator, periodically observed every three minutes. The pointing was checked on the gain calibrator in X-band every 30 minutes. The calibrated data were concatenated and imaged using Briggs weighting with a robust parameter of 0.5 . Deconvolution using CASA imfit reveals that the source is marginally resolved. The flux calibration uncertainty is $10 \%$.

Observations at $680 \mathrm{GHz}(440 \mu \mathrm{m})$ were obtained using ALMA in Cycle 2 in 2014 July in the C34-3 configuration with Band 9 (Baryshev et al. 2015) as part of program 2013.1.00100.S. The observations were taken in four spectral windows of 1920 channels: three windows have a bandwidth of 937.5 MHz, centered on 661,659 , and $675 \mathrm{GHz}$, the fourth spectral window was centered on $672 \mathrm{GHz}$ with a bandwidth of $1875 \mathrm{MHz}$. The total continuum bandwidth was $\sim 4.7 \mathrm{GHz}$. The flux was calibrated using J1517-243, the bandpass with
J1427-4206 and the phase with J1626-2951. The total onsource integration time was 52 minutes. The data were selfcalibrated and imaged using Briggs weighting with a robust parameter of 0.5 . The flux calibration uncertainty is $20 \%$.

Table 1 lists the properties of the images. The astrometric accuracy is set by the calibrators, and is typically $\precsim 30$ mas for these two data sets, which is much smaller than the beam size.

\section{RESULTS}

Figure 1 shows images of the dust continuum emission at 680 and $34 \mathrm{GHz}$. The $680 \mathrm{GHz}$ continuum has a similar asymmetry as observed in van der Marel et al. (2013) in the Band 9 Cycle 0 data, though these new data have improved angular resolution. The $34 \mathrm{GHz}$ continuum emission peaks at the same location, but is much more azimuthally concentrated. This is not a sensitivity effect: when the $680 \mathrm{GHz}$ data are restored with the same cleaning beam as the $34 \mathrm{GHz}$ data, its azimuthal extent is still clearly wider than in the measured $34 \mathrm{GHz}$ data (see Figure 1(d)). The radial width cannot be compared due to the vertically elongated VLA beam shape, which is caused by the low declination of the source with respect to the VLA site, but given the disk geometry, this elongation does not affect constraints on the azimuthal width, the key parameter of interest here. Spatial filtering can be ruled out as an explanation for the different azimuthal extents; these data recover all of the flux found on the shortest baselines from previous VLA observations in the DnC configuration (beam size 3 ". $3 \times 1 " .3$, flux $=252 \pm 11 \mu \mathrm{Jy})$. Also, we have simulated the ALMA image as if observed by the VLA by sampling the ALMA image on the VLA visibilities (Figure 1(e)) to rule out horizontal spatial filtering. Besides, the largest angular scales recovered by the $\mathrm{CnB}$ observation is $5^{\prime \prime}$, which is much larger than the disk. Thus, the centimeter-sized dust grains (traced by the $34 \mathrm{GHz}$ continuum) have a narrower azimuthal distribution than the millimeter-sized dust grains (traced by the $690 \mathrm{GHz}$ 
continuum). The peak brightness temperatures for the $680 \mathrm{GHz}$ and $34 \mathrm{GHz}$ are 31 and $1 \mathrm{~K}$, respectively.

In order to quantify the concentration of the dust grains, the morphology of the continuum image is fit to a two-dimensional fourth power Gaussian intensity profile $I(r, \phi)$ (van der Marel et al. 2013):

$$
I(r, \phi)=I_{c} \exp \left(\frac{-\left(r-r_{c}\right)^{4}}{2 r_{w}^{4}}\right) \exp \left(\frac{-\left(\phi-\phi_{c}\right)^{4}}{2 \phi_{w}^{4}}\right) .
$$

Previous studies describe azimuthal dust asymmetries due to vortices as regular second power Gaussians (Lyra \& Lin 2013; Pérez et al. 2014), but it was found that our data are fitted much better with the fourth power equation. The stellar position was set to $16^{\mathrm{h}} 27^{\mathrm{m}} 37^{\mathrm{s}} .185-24^{\circ} 30^{\prime} 35^{\prime \prime}$. 39 , taken from a Keplerian model fit to the $\mathrm{CO}$ isotopologue data from the same ALMA data set (van der Marel et al. 2015b). The proper motion is negligible compared to the beam size for the period in between these observation sets ( $\sim 6$ months), so they can be overlaid directly. For the fitting, we use $\chi^{2}$ minimization over a grid of $\phi_{c}, \phi_{w}, r_{c}$ and $r_{w}$ with steps of $1^{\circ}$ and 1 AU for angle and radius respectively. The fitting is performed in the $u v$-plane, with the model visibilities sampled onto the observed spatial frequencies. We find the best fit (see Figure 2) for $r_{c}=61( \pm 2) \mathrm{AU}$, $r_{w}=14( \pm 2) \mathrm{AU}, \phi_{c}=100( \pm 3)^{\circ}$ with $\phi_{w}=41( \pm 4)^{\circ}$ for the $680 \mathrm{GHz}$ and $\phi_{w}=18( \pm 7)^{\circ}$ for the $34 \mathrm{GHz}$ continuum. The area at $680 \mathrm{GHz}$ is smaller than that reported in van der Marel et al. (2013), due to better spatial resolution, although still within the derived error bars. The values and errors for $r_{c}, r_{w}$, and $\phi_{c}$ are mainly constrained by the ALMA data, as the VLA data did not have sufficient spatial resolution to constrain the error bars. The errors are estimated by rescaling the reduced $\chi^{2}$ to 1 .

The important conclusion is that the azimuthal width of the centimeter emission is $2.3( \pm 0.9)$ times narrower than the millimeter emission. The aspect ratio of the submillimeter emission is $3.1( \pm 0.6)$, and $>1.4$ for the centimeter emission.

The residual of the $680 \mathrm{GHz}$ image still shows significant emission outside of the Gaussian fit (peak $25 \sigma$ ), especially at the tail in the east: $15 \%$ of the total absolute flux remains in the residual. The intensity equation described by Equation (1) is clearly not sufficient to describe the detailed structure: additional (vertical) features may be present. The disk is known to have a large scale height (Bruderer et al. 2014), which is not taken into account in the intensity model, while the dust is possibly optically thick at this wavelength and the vertical structure may be relevant. However, more than $85 \%$ of the structure is recovered by the Gaussian intensity equation and the SNR is very high, the description is sufficient to compare the azimuthal width at the two wavelengths.

The residual of the $34 \mathrm{GHz}$ image clearly shows a point source at the stellar position, with a peak flux of $36 \mu \mathrm{Jy}$ beam $^{-1}$ $(10 \sigma)$. This point source can be either dust emission from an unresolved inner disk, or free-free/synchrotron emission from ionized gas close to the star. Since this emission is not seen in the ALMA data, the spectral index of this emission is $<1.3$, so dust emission is unlikely. The origin of this emission can be determined using longer wavelength observations (Rodmann et al. 2006; Ubach et al. 2012). The total flux of the dust emission in the dust trap is thus only $216 \mu \mathrm{Jy}$ at $34 \mathrm{GHz}$.
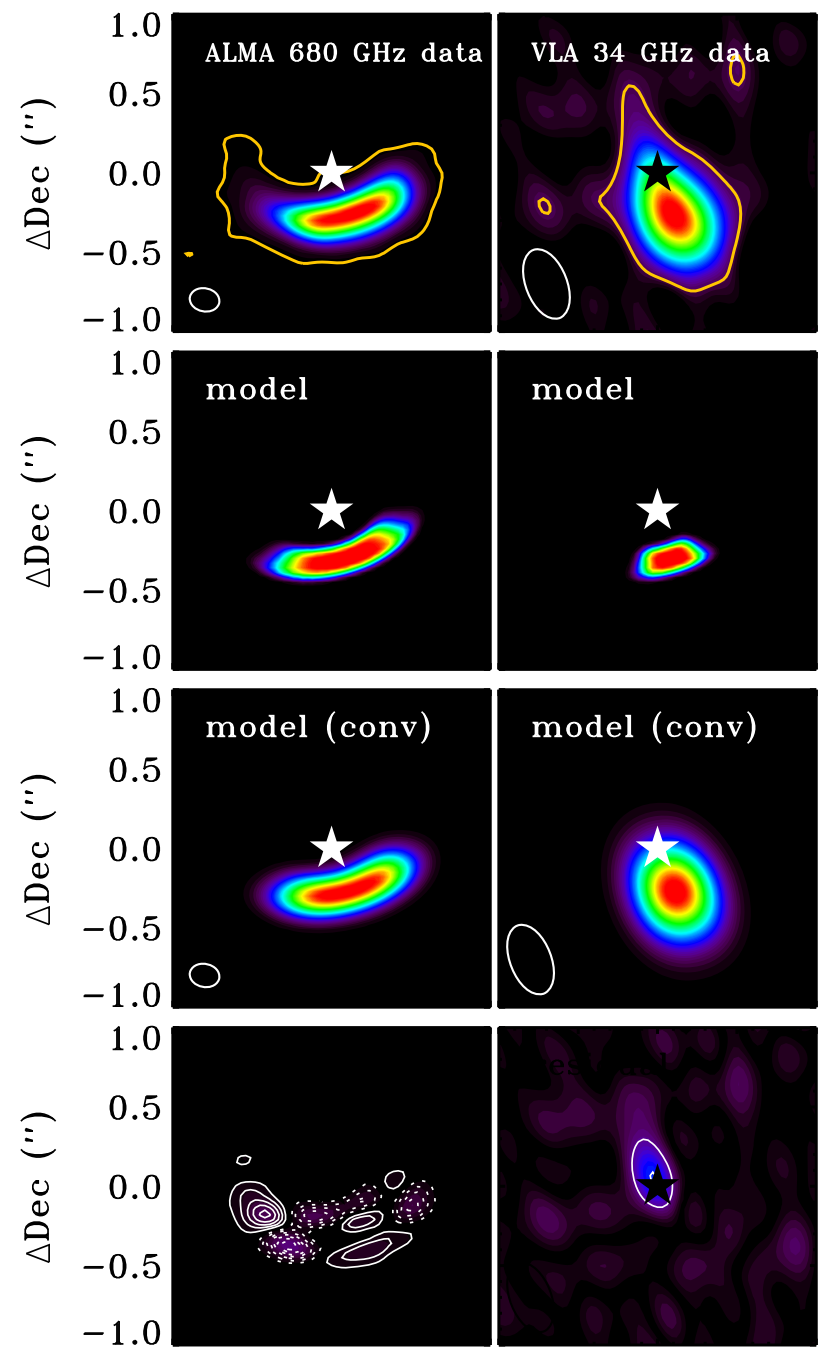

$$
\begin{array}{ccc}
1.0 & 0.5 \quad 0.0-0.5-1.0 & 0.5 \quad 0.0-0.5-1.0 \\
\triangle \mathrm{RA}(") & \triangle \mathrm{RA}\left({ }^{\prime \prime}\right)
\end{array}
$$

Figure 2. Best fits of the intensity profile given in Equation (1) to the dust continuum for the $680 \mathrm{GHz}$ (left) and $34 \mathrm{GHz}$ (right) data. The top panel shows the data in colorscale (the yellow contour shows the $3 \sigma$ level), the middle two panels the unconvolved and convolved model, and the bottom panel the residual image, with the absolute values of the residual in the same colorscale as the data image, and overlaid contours at $5 \sigma$ spacing $\left(\sigma_{680 \mathrm{GHz}}\right.$ $=0.6 \mathrm{mJy}_{\text {beam }}^{-1}, \sigma_{34 \mathrm{GHz}}=3.5 \mu \mathrm{Jy}$ beam $^{-1}$ ). Dashed contours indicate negative intensity levels.

\section{DISCUSSION AND SUMMARY}

The difference in azimuthal width between the two continuum images suggests a segregation of particle sizes, where centimeter-sized grains are more azimuthally concentrated than millimeter-sized dust grains. However, optical depth effects can hide a narrow concentration of millimeter-dust grains equivalent to the $34 \mathrm{GHz}$ morphology on top of the apparent millimeter-dust distribution. The optical depth $\tau_{680 \mathrm{GHz}}$ cannot be measured independently from the temperature with the available data. The measured brightness temperature at $680 \mathrm{GHz}$ of $31 \mathrm{~K}$ (assuming the Planck equation) is only a factor of two lower than the calculated physical dust temperature of $60 \mathrm{~K}$ (at 60 AU radius in a physical disk model, Bruderer et al. 2014) so the emission is likely not highly optically thick. In order to quantify the optical depth at $680 \mathrm{GHz}$, we assume that the peak millimeter emission 


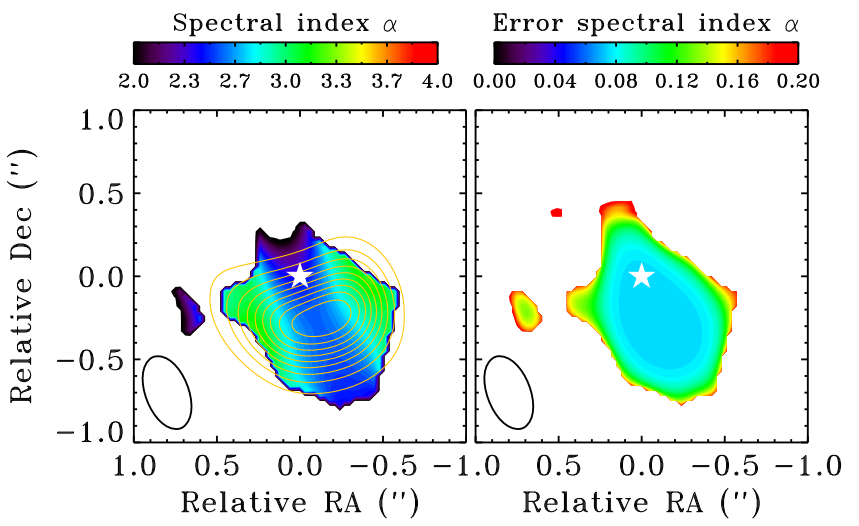

Figure 3. Comparison of the ALMA and VLA data of Oph IRS 48, imaged with the same synthesized beam of 0 ". $46 \times 0$ ". 26 (lower left corner). The left plot shows the spatially resolved spectral index $\alpha$, using 34 and $680 \mathrm{GHz}$ data. The right plot shows the error on $\alpha$ as a function of position. The ALMA data are overlaid in yellow contours with $10 \%, 20 \%, \ldots, 90 \%$ of the peak flux. The spectral index decreases at the peak of the dust emission. Data points where the $34 \mathrm{GHz}$ flux density is less than $2 \sigma(\sigma=3.5 \mu \mathrm{Jy})$ are excluded.

originates from an isothermal area $\Omega_{\text {beam }}(\mathrm{sr})$ of the beam size with $T_{\text {dust }}=60 \mathrm{~K}$. This is a conservative limit since $\tau$ likely decreases azimuthally outward. For the peak flux of $189 \mathrm{mJy}$, $\tau_{680 \mathrm{GHz}}=0.5$, so the emission is indeed marginally optically thick at $680 \mathrm{GHz}$. If the dust temperature is much lower than assumed here, the emission becomes fully optically thick and a narrow concentration of millimeter-dust grains could remain hidden. Locally lowering the temperature is possible by shadowing due to an inclined inner disk, such as proposed for HD142527 (Marino et al. 2015) to explain the scattered light emission. For Oph IRS 48, there is no evidence for a local temperature drop, so we propose that the difference in emission originates from a spatial segregation between particles, where the centimeter-sized grains are more concentrated than the millimeter-sized grains. Spatially resolved continuum observations at intermediate wavelengths are required to confirm the dust temperature and optical depth.

Combining the total $34 \mathrm{GHz}$ flux of $216 \pm 22 \mu \mathrm{Jy}$ with SMA and disk integrated ALMA observations at 230, 345, and $680 \mathrm{GHz}$ gives a spectral slope of $\alpha=2.84 \pm 0.06\left(F_{\nu} \sim \nu^{\alpha}\right)$. The fluxes are $50 \pm 7.5,160 \pm 24$, and $1000 \pm 200 \mathrm{mJy}$, respectively, taken from Brown et al. (2012) and this work, with the errors dominated by the flux calibration uncertainty. Measuring $\alpha$ independently between 680 and $230 \mathrm{GHz}$ $(2.8 \pm 0.2)$ and between 230 and $34 \mathrm{GHz}(2.85 \pm 0.7)$ results in the same value within error bars, supporting at most modest optical depth at $680 \mathrm{GHz}$.

Because both the VLA and the ALMA data are spatially resolved, the spectral index can be computed as a function of position (see Figure 3). For this figure, the ALMA data were convolved with the same synthesized beam as the VLA observations. The figure shows that $\alpha$ increases from $2.6 \pm$ 0.08 in the center to $3.3 \pm 0.15$ in the outer wings, similar to the synthetic spectral index map from Birnstiel et al. (2013) of azimuthal trapping. The error in spectral index across the image is calculated based on the signal-to-noise ratio, as shown in the right panel of Figure 3. Therefore, the decrease of the spectral index at the peak of the dust emission is significant. Due to the flux calibration uncertainty, which is $20 \%$ for ALMA Band 9 and $10 \%$ for the VLA Ka band observations, the calibration error over the entire image on $\sigma_{\alpha}$ is $\sim 0.07$, but this does not vary with position and therefore it is not included in the error plot.

Analysis of the dust opacity index $\beta\left(\tau_{\nu} \propto \nu^{\beta}\right)$ is an important tool in constraining the dust particle sizes in the disk. When the dust emission is optically thin $(\tau \ll 1)$ and in the Rayleigh-Jeans regime, $\beta=\alpha-2$, where $\beta<1$ indicates dust growth to larger than millimeter-sized particles (Draine 2006). For IRS 48, the $680 \mathrm{GHz}$ is not optically thin and these assumptions cannot be made. Therefore, the dust opacity index thus has to be derived from the optical depth $\tau_{\nu}$ itself.

Using $\tau_{\nu} \propto \nu^{\beta}, \beta$ can be calculated as a function of position by calculating $\tau_{\nu}$ at both frequencies at different positions, assuming $T_{\text {dust }}=60 \mathrm{~K}$ and the flux filling the beam. We find $\beta \sim 0.7 \pm 0.1$ in the center and $\beta \sim 1.3 \pm 0.3$ in the outer parts for a uniform temperature. As the continuum peak is likely more optically thick in the center, the emission there traces higher vertical layers where the temperature is higher, implying an even lower $\beta$ : for $T_{\text {dust }}=200 \mathrm{~K}$ in the center, $\beta=0.6 \pm 0.1$ according to the same calculation. The azimuthal trend in $\beta$ is thus consistent with increased dust growth (increase of $a_{\max }$ ) or an increase of larger particles in the center (increase of $a_{\mathrm{min}}$ ). On the other hand, if the temperature is in fact as low as $31 \mathrm{~K}, \beta$ would increase in the center. In order to get a uniform $\beta$ along the entire azimuthal shape (which implies no change in dust growth or size segregation), $T_{\text {dust }}$ needs to be as low as $24 \mathrm{~K}$ at the center, which is more than a factor of two lower than the derived midplane dust temperature (Bruderer et al. 2014), while the optically thick emission likely originates from higher vertical layers with even higher temperatures. Thus, within reasonable azimuthal variations of the temperature, the results hint at a variation in $\beta$, though some uncertainties remain.

In summary, the centimeter emission provides further support for the dust trapping mechanism in the Oph IRS 48 disk: within the assumptions of the $680 \mathrm{GHz}$ optical depth and the temperature field, the centimeter-sized grains appear to be more concentrated in azimuth than the millimeter-sized grains as predicted in analytical dust models of azimuthal pressure maxima (Birnstiel et al. 2013; Lyra \& Lin 2013). Inside the dust trap, grains may have reached centimeter sizes (and perhaps even larger), the first step toward planetesimal and planet formation.

We are grateful to E. F. van Dishoeck and M. Schmalzl for useful discussions, to the referee for useful comments and to the VLA staff for their efforts to observe this program after the configuration move. N.M. is supported by the Netherlands Research School for Astronomy (NOVA). J.T. acknowledges support from grant 639.041.439 from the Netherlands Organisation for Scientific Research (NWO). T.B. acknowledges support from NASA Origins of Solar Systems grant NNX12AJ04G. Astrochemistry in Leiden is supported by the Netherlands Research School for Astronomy (NOVA), by a Royal Netherlands Academy of Arts and Sciences (KNAW) professor prize, and by the European Union A-ERC grant 291141 CHEMPLAN. The National Radio Astronomy Observatory is a facility of the National Science Foundation operated under cooperative agreement by Associated Universities, Inc. This paper makes use of the following ALMA data: ADS/ JAO.ALMA/2013.1.00100.S. ALMA is a partnership of ESO (representing its member states), NSF (USA), and NINS 
(Japan), together with NRC (Canada) and NSC and ASIAA (Taiwan), in cooperation with the Republic of Chile. The Joint ALMA Observatory is operated by ESO, AUI/NRAO, and NAOJ.

Facilities: ALMA, EVLA.

\section{REFERENCES}

Ataiee, S., Pinilla, P., Zsom, A., et al. 2013, A\&A, 553, L3

Barge, P., \& Sommeria, J. 1995, A\&A, 295, L1

Baryshev, A. M., Hesper, R., Mena, F. P., et al. 2015, A\&A, 577, A129

Birnstiel, T., Dullemond, C. P., \& Brauer, F. 2010, A\&A, 513, A79

Birnstiel, T., Dullemond, C. P., \& Pinilla, P. 2013, A\&A, 550, L8

Brauer, F., Dullemond, C. P., \& Henning, T. 2008, A\&A, 480, 859

Brown, J. M., Rosenfeld, K. A., Andrews, S. M., Wilner, D. J., \& van Dishoeck, E. F. 2012, ApJL, 758, L30

Bruderer, S., van der Marel, N., van Dishoeck, E. F., \& van Kempen, T. A. 2014, A\&A, 562, A26

Casassus, S., van der Plas, G., M, S. P., et al. 2013, Natur, 493, 191

Draine, B. T. 2006, ApJ, 636, 1114

Espaillat, C., Muzerolle, J., Najita, J., et al. 2014, in Protostars and Planets VI, ed. H. Beuther et al. (Tucson, AZ: Univ. Arizona Press), 497

Flock, M., Ruge, J. P., Dzyurkevich, N., et al. 2015, A\&A, 574, A68

Follette, K. B., Grady, C. A., Swearingen, J. R., et al. 2015, ApJ, 798, 132

Fukagawa, M., Tsukagoshi, T., Momose, M., et al. 2013, PASJ, 65, L14
Fung, J., Shi, J.-M., \& Chiang, E. 2014, ApJ, 782, 88

Geers, V. C., Pontoppidan, K. M., van Dishoeck, E. F., et al. 2007, A\&A, 469, L35

Johansen, A., Youdin, A., \& Klahr, H. 2009, ApJ, 697, 1269

Klahr, H. H., \& Henning, T. 1997, Icar, 128, 213

Lyra, W., \& Lin, M.-K. 2013, ApJ, 775, 17

Marino, S., Perez, S., \& Casassus, S. 2015, ApJL, 798, L44

Pérez, L. M., Isella, A., Carpenter, J. M., \& Chandler, C. J. 2014, ApJL, 783, L13

Perez, S., Casassus, S., Ménard, F., et al. 2015, ApJ, 798, 85

Pinilla, P., Birnstiel, T., Ricci, L., et al. 2012, A\&A, 538, A114

Pinilla, P., de Juan Ovelar, M., Ataiee, S., et al. 2015, A\&A, 573, A9

Raettig, N., Lyra, W., \& Klahr, H. 2013, ApJ, 765, 115

Rodmann, J., Henning, T., Chandler, C. J., Mundy, L. G., \& Wilner, D. J. 2006, A\&A, 446, 211

Ubach, C., Maddison, S. T., Wright, C. M., et al. 2012, MNRAS, 425, 3137

van der Marel, N., van Dishoeck, E., Bruderer, S., et al. 2015b, A\&A submitted

van der Marel, N., van Dishoeck, E., Bruderer, S., Perez, L. M., \& Isella, A. 2015a, A\&A, 579, 106

van der Marel, N., van Dishoeck, E. F., Bruderer, S., et al. 2013, Sci, 340, 1199

Weidenschilling, S. J. 1977, MNRAS, 180, 57

Whipple, F. L. 1972, in From Plasma to Planet, ed. A. Elvius (New York: Wiley-Interscience), 211

Windmark, F., Birnstiel, T., Güttler, C., et al. 2012, A\&A, 540, A73

Zhang, K., Isella, A., Carpenter, J. M., \& Blake, G. A. 2014, ApJ, 791, 42

Zhu, Z., \& Stone, J. M. 2014, ApJ, 795, 53 